\title{
Suppression of human metapneumovirus (HMPV) infection by the innate sensing gene CEACAM1
}

\author{
Mohammad Diab ${ }^{1, *}$, Alon Vitenshtein ${ }^{1, *}$, Yaron Drori ${ }^{2,3}$, Rachel Yamin ${ }^{1}$, Oded \\ Danziger ${ }^{4}$, Rachel Zamostiano ${ }^{4}$, Michal Mandelboim ${ }^{2,3}$, Eran Bacharach ${ }^{4}$ and Ofer \\ Mandelboim ${ }^{1}$ \\ ${ }^{1}$ The Lautenberg Center for General and Tumor Immunology, The BioMedical Research Institute Israel Canada of the Faculty \\ of Medicine, The Hebrew University Hadassah Medical School, Jerusalem, Israel \\ ${ }^{2}$ Central Virology Laboratory, Ministry of Health, Public Health Services, Chaim, Sheba Medical Center, Tel Hashomer, Ramat- \\ Gan, Israel \\ ${ }^{3}$ Department of Epidemiology and Preventive Medicine, School of Public Health, Sackler Faculty of Medicine, Tel-Aviv \\ University, Tel-Aviv, Israel \\ ${ }^{4}$ Department of Cell Research and Immunology, Faculty of Life Sciences, Tel Aviv University, Tel Aviv, Israel \\ * These authors have equally contributed to this work \\ Correspondence to: Ofer Mandelboim, email: oferm@ekmd.huji.ac.il \\ Keywords: HMPV, CEACAM1, PRRs, PAMPs, RLRs, Immunology and Microbiology Section, Immune response, Immunity \\ Received: July 18, $2016 \quad$ Accepted: September 01, $2016 \quad$ Published: September 12, 2016
}

\section{ABSTRACT}

The innate sensing system is equipped with PRRs specialized in recognizing molecular structures (PAMPs) of various pathogens. This leads to the induction of anti-viral genes and inhibition of virus growth. Human Metapneumovirus (HMPV) is a major respiratory virus that causes an upper and lower respiratory tract infection in children. In this study we show that upon HMPV infection, the innate sensing system detects the viral RNA through the RIG-I sensor leading to induction of CEACAM1 expression. We further show that CEACAM1 is induced via binding of IRF 3 to the CEACAM1 promoter. We demonstrate that induction of CEACAM1 suppresses the viral loads via inhibition of the translation machinery in the infected cells in an SHP2dependent manner. In summary, we show here that HMPV-infected cells upregulates CEACAM1 to restrict HMPV infection.

\section{INTRODUCTION}

HMPV, discovered in 2001, is classified as the first human member of the Metapneumovirus genus of the paramyxovirus family [1]. HMPV is a ubiquitous respiratory pathogen, which is known to have been circulating in human populations for decades [1]. Several studies showed that HMPV is a leading cause of upper and lower respiratory tract infections in children and immunocompromised patients [2-12]. The clinical symptoms of patients infected with HMPV range from mild symptoms to severe bronchiolitis and pneumonia [5, 13-15], which can lead to death $[16,17]$.

Viruses contain conserved molecular structures known as PAMPs, which are recognized by several families of innate receptors, collectively named PRRs [1823]. One important class of such receptors are the retinoic acid-inducible gene (RIG)-I-like receptors (RLRs) [18, 23,
24]. RLRs are intracellular sensors of viral components that include single stranded RNA (ssRNA) and double stranded RNA (dsRNA). Upon recognition of such PAMPs by the PRRs, different signaling pathways are activated leading to transcription of a multitude of antiviral genes $[18,24]$.

In epithelial cells, the innate immune sensor RIG-I was shown to sense HMPV infection leading to the secretion of type I IFNs through interferon response factor 7 (IRF7) and interferon response factor 3 (IRF3) [25-28]. Inhibition of RIG-I expression significantly decreases the production of type I IFN, pro-inflammatory cytokines and chemokines [29].

Carcinoembryonic antigen-related cell adhesion molecule 1 (CEACAM1) belongs to the carcinoembryonic antigen (CEA) family [30, 31]. It binds homophilically or heterophilically to members of the CEACAM family [32]. CEACAM1 is primarily an 
inhibitory receptor which delivers its inhibitory signal via tyrosine based inhibitory motifs (ITIMs), through the tyrosine phosphatase (SHP1) in immune cells and (SHP2) in non-immune cells [32-36]. In the current study, we show that following HMPV infection, CEACAM1 is induced by the innate sensing system to control viral production in an SHP2-dependent manner.

\section{RESULTS}

\section{HMPV infection induces cell surface expression of CEACAM1}

To investigate whether HPMV infection affects the expression of various natural killer $(\mathrm{NK})$ cell ligands, we infected A549 cells with HMPV. 48 hours after the infection we validated that the cells were indeed infected by using qRT-PCR (Figure 1a) or by infection with recombinant HMPV virus expressing green fluorescent protein GFP (HMPV/GFP) [41] (Figure 1b). We next stained the mocked-infected and the infected cells for the expression of various immune ligands such as CEACAM1 (Figure 1c and 1d, respectively). Practically 100\% of the cells were infected, as indicated in Figure 1d. We observed a significant induction of CEACAM1 expression following HMPV infection (Figure 1d and 1e). Analysis of expression kinetics of CEACAM1 showed that protein expression was visible as early as 6 hours post infection, peaking at 12 hours post infection (Figure 1f). Elevation of CEACAM1 mRNA was also noticed following HMPV infection (Figure 1g).

To test whether the HMPV-mediated CEACAM1 induction has functional consequences we used a cellbased reporter assay that employs the murine $\mathrm{BW}$ thymoma cells. For this assay, the BW cells were transfected with a construct that is composed of a chimeric protein in which the extracellular portion of CEACAM1 receptor is fused to the transmembrane and tail domain of the CD3z chain (Figure 2a). This system triggers IL-2 secretion upon binding of CEACAM1 to its ligand (which is CEACAM1). We infected A549 cells with HMPV and 48 hours later, we incubated the infected cells with BW/ CEACAM1. As can be seen, significant induction of IL-2 secretion was observed following HMPV infection (Figure 2b). Blocking of the CEACAM1 interaction abrogated this induction, indicating that the induced IL-2 secretion resulted from homophilic CEACAM1-CEACAM1 interaction (Figure 2b).

\section{CEACAM1 expression is induced by the innate immune cellular RIGI-IRF3 sensing system}

We next proceeded to study the mechanism of HMPV-mediated induction of CEACAM1. Initially, we observed that a viable virus is required to induce CEACAM1 expression, since CEACAM1 was not induced following infection with UV-inactivated virus (Figure 3a). To investigate whether HMPV RNA can induce CEACAM1 expression we isolated the HMPV RNA and transduced it to A549 cells. As can be seen in Figure 3b, viral RNA, UV-treated or not, induced the expression of CEACAM1. Finally, we investigated whether the RNAmediated induction of CEACAM1 is specific to the viral RNA only. For this we transfected the A549 cells with Polyinosinic-polycytidylic acid (PolyI:C), a synthetic analog of double-stranded RNA and observed that PolyI:C transfection, UV-treated or not, also induce CEACAM1 expression (Figure 3c). These observations suggest that the cellular nucleic acid sensing system is responsible for the induction of CEACAM1.

Previous studies have shown that RIG-I is the sensor of HMPV RNA [26]. We next tested whether RIG-I, via its mediator IRF-3, is responsible for the CEACAM1 induction. For this, we knocked-down RIG-I (Figure 4a) and IRF3 (Figure 4b) by using short hairpin RNAs (shRNAs). As can be seen, knockdowns of both proteins almost completely abolished the upregulation of CEACAM1 following 48 hours of HMPV infection, compared to the control (Figure 4c). This finding demonstrates that the innate immune RNA sensor, RIG-I, leads to the induction of CEACAM1 through the RIGIIRF3 pathway.

The CEACAM1 promoter contains a predicted IRF3 binding site (Figure 5). To test whether this site mediated the CEACAM1 induction during HMPV infection, we used a luciferase reporter assays in which $600 \mathrm{bp}$ of the wild-type or IRF3 binding site mutated (IRF3 Mut) promoter sequences was fused to luciferase (Figure 5a and $5 \mathrm{~b}$ respectively). While the CEACAM1 promoter sequence mediated a strong induction of luciferase activity, following 48 hours of HMPV infection, mutation in the predicted IRF3 binding site, significantly decreased the luciferase activity (Figure 5c).

\section{CEACAM1 suppresses HMPV virus production by inhibiting protein synthesis in an SHP2- dependent manner}

We next proceeded to understand why CEACAM1 expression is induced following HMPV infection. Homophilic binding of CEACAM1 transmits an inhibitory signal via the SHP2 phosphatase in non-immune cells. We initially knocked-down either CEACAM1 (Figure 6a, qRT-PCR verification in b) or SHP2 (Figure 6c qRT-PCR verification in d), in A549 cells by using specific shRNAs. Twenty four hours following HMPV infection, viral loads were quantified. As can be seen, a significant elevation in viral loads was observed in cells expressing the CEACAM1 and SHP2 specific shRNAs compared to the 
a

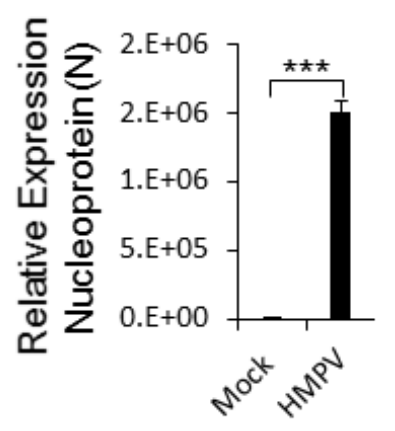

C

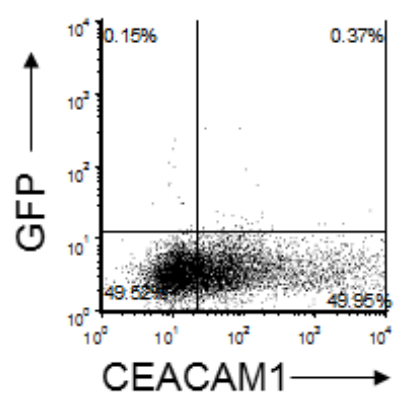

b

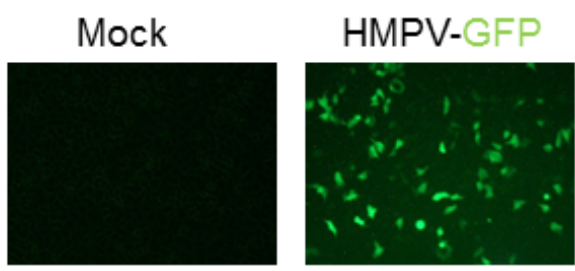

d

HMPV-GFP

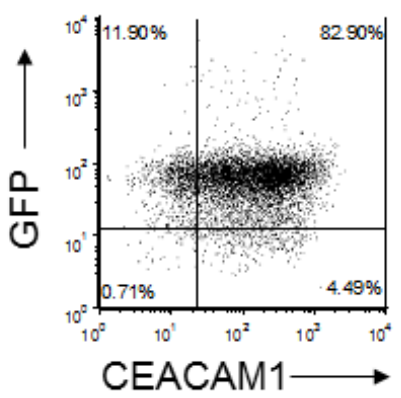

e

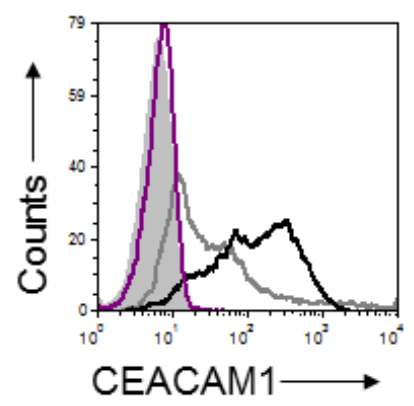

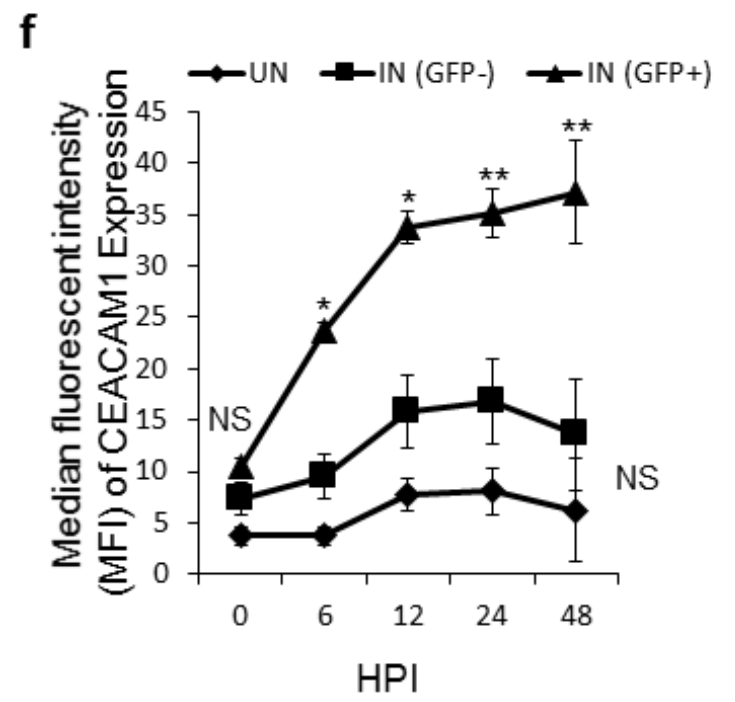

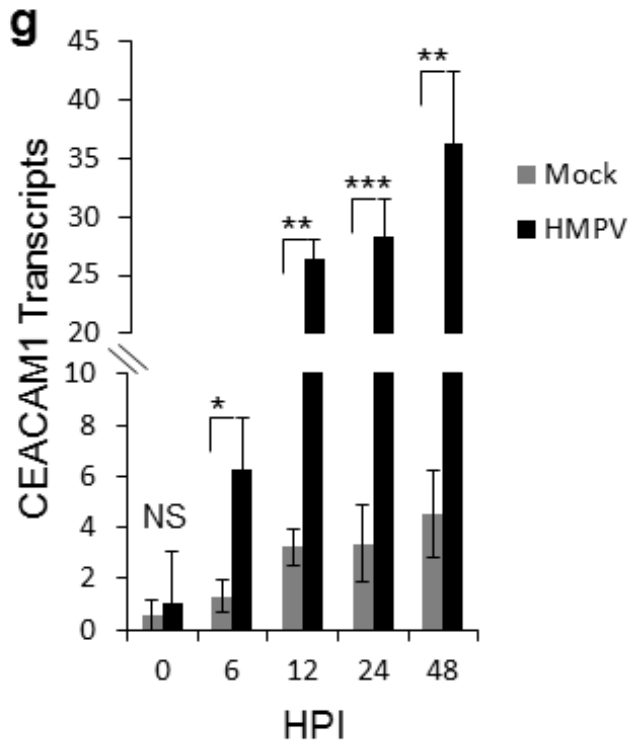

Figure 1: Expression of CEACAM1 in A549 cell line following HMPV infection. a. qRT-PCR of the HMPV nucleoprotein (N) gene of HMPV in A549 infected cells (HMPV). The expression of nucleoprotein N in HMPV infected cells was determined relative to mock treated cells (Mock), whose expression level was set as 1. b. Fluorescent microscopy of mock treated (Mock) and A549 cells infected with the recombinant HMPV expressing GFP protein (HMPV-GFP). c.-d. Dot plots of CEACAM1 staining and GFP expression (indicative of virus infection) of mock (c) and HMPV-infected A549 cells (d). e. FACS analysis of CEACAM1 expression shown in (c and d) on the mock treated A549 cells (empty gray histogram) and on HMPV-infected A549 cells (empty black histogram), at 48h post infection. The filled gray histogram and the empty purple histogram represent the staining of the mock treated and infected A549 cells with control antibody, respectively. f. The expression of CEACAM1 was monitored on infected cells (IN (GFP+), on the uninfected cells that were present in the same culture IN (GFP-) and on parental A549 cells that were mock-treated (UN). The expression of CEACAM1 is presented as median fluorescent intensity (MFI) and is shown at various hours post infection (HPI). g. Real time PCR quantification of CEACAM1 expression on the mock-treated (Mock) and the HMPV infected cells (HMPV) during the indicated time points (HPI). NS, nonsignificant. Values are shown as means $\pm \mathrm{SEM}$. The figure shows data from two experiments combined. ${ }^{* * *} p<0.01,{ }^{* *} p<0.03,{ }^{*} p<0.05$. 
control (Figure 6e). To further corroborate these results we overexpressed the full CEACAM1 gene or two mutants of CEACAM1: 1) CEACAM1 lacking the intracellular ITIM containing signaling domain (CEACAM1 Mut) and 2) Fusion of the intracellular signaling domain to GFP (GFP-ITIM) (Figure 6f). Following 24 hours of HMPV infection, significant elevation of viral loads was observed in cells expressing CEACAM1 Mut or GFP-ITIM, as compared to cells expressing the full length CEACAM1 gene (Figure 6g). Additionally, we over-expressed the SHP2 phosphatase in A549 cells (Figure 6h). Following 24 hours of HMPV infection, we observed a significant decrease in viral loads in cells over-expressing SHP2 compared to control (Figure 6i). Finally, we proceeded to investigate the mechanism by which CEACAM1 and its phosphatase SHP2 inhibit the HMPV production. We assessed protein production in A549 cells expressing shRNAs against CEACAM1 and SHP2. The treated cells were labeled with ${ }^{35} \mathrm{~S}-$ Methionine and following 24 hours of HMPV infection the ${ }^{35} \mathrm{~S}$-Methionine labelling was determined. As can be seen, cells expressing shRNAs targeting CEACAM1 or SHP2, exhibited about a 3 fold increase in ${ }^{35} \mathrm{~S}-$ Methionine incorporation following infection, as compared to the scrambled shRNA (Figure $6 \mathrm{j})$. Thus, we conclude that CEACAM1 suppresses HMPV infection in an SHP2-dependent manner by inhibiting the protein production machinery.

\section{DISCUSSION}

We know today that practically all nucleated cells participate in innate recognition of viruses. They all contain cellular sensors (PRRs) that detect PAMPs, among them are viral nucleic acids. This leads to the production of IFNs, antiviral genes and activation of signaling cascades which results in pathogens inhibition [18-20, 24, 42].

We recently demonstrated that upon sensing of influenza by RIG-I and HCMV by IFI16, CEACAM1 expression is induced [43]. Here we show that RIG-I sensing of HMPV also leads to CEACAM1 induction and to the establishment of an anti-viral state in the infected cells. We suggest the following (Figure 7): 1) Upon HMPV infection, the negative single stranded RNA of HMPV is exposed in the cytoplasm. 2) RIG-I senses viral RNA 3) IRF3 is activated, and translocate into the nucleus where it binds the CEACAM1 promoter. 4) CEACAM1 transcription is induced. 5) CEACAM1 protein is expressed. 6) The hemophilic binding of CEACAM1 leads to activation of SHP2 phosphatase which in turns leads to inhibition in the cellular translation and to viral suppression. a

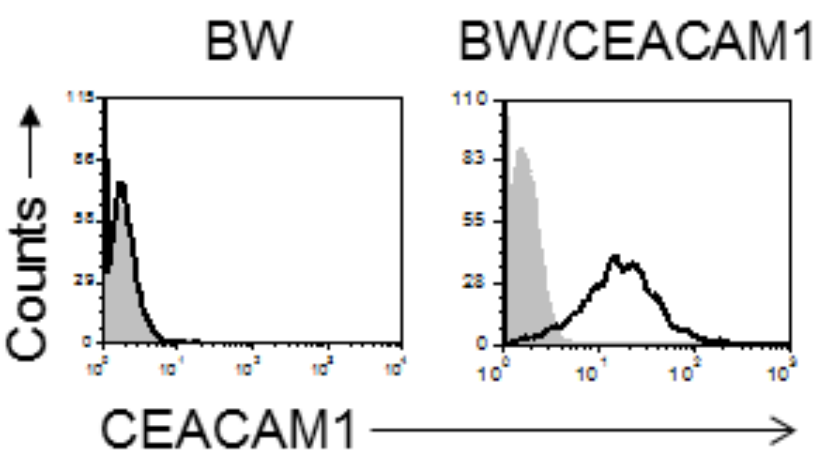

b

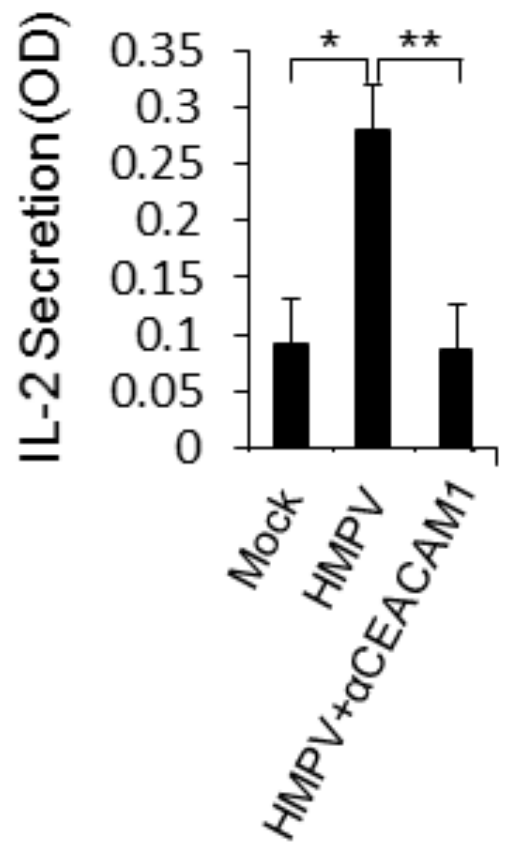

Figure 2: The expression of CEACAM1 on HMPV-infected A549 cells is functional. a. FACS analysis of parental BW cells and BW cells transfected to expressed a chimeric protein composed of the extracellular portion of CEACAM1 fused to mouse zeta chain (BW/CEACAM1). The filled gray histogram is the background staining and the empty black histogram is the staining of CEACAM1. b. IL-2 secretion from the BW/CEACAM1 cells following $48 \mathrm{~h}$ incubation with the indicated A549 cells that were either mock infected (Mock), infected with HMPV (HMPV) or infected with HMPV and blocked with anti CEACAM1 mAb (HMPV+ + CEACAM1). Values are shown as means $\pm \mathrm{SEM}$. The figure shows data from three experiments combined. ${ }^{*} p<0.05, * * p<0.02$. 
a

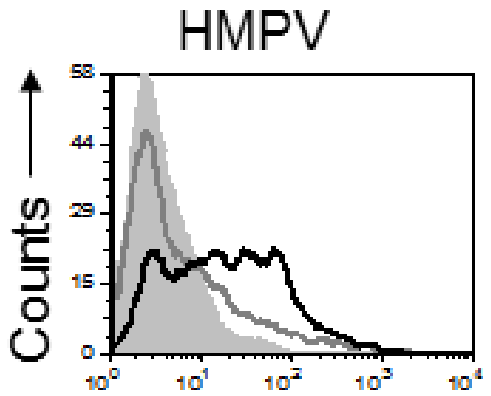

CEACAM1

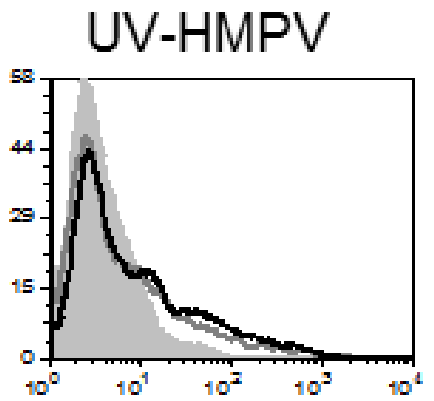

b

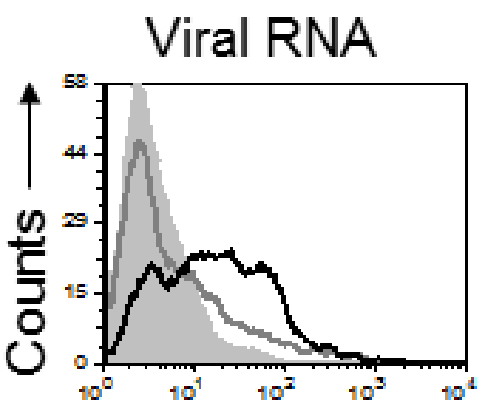

CEACAM1

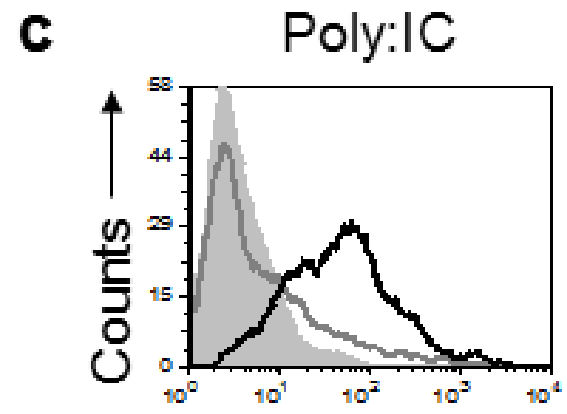

CEACAM1
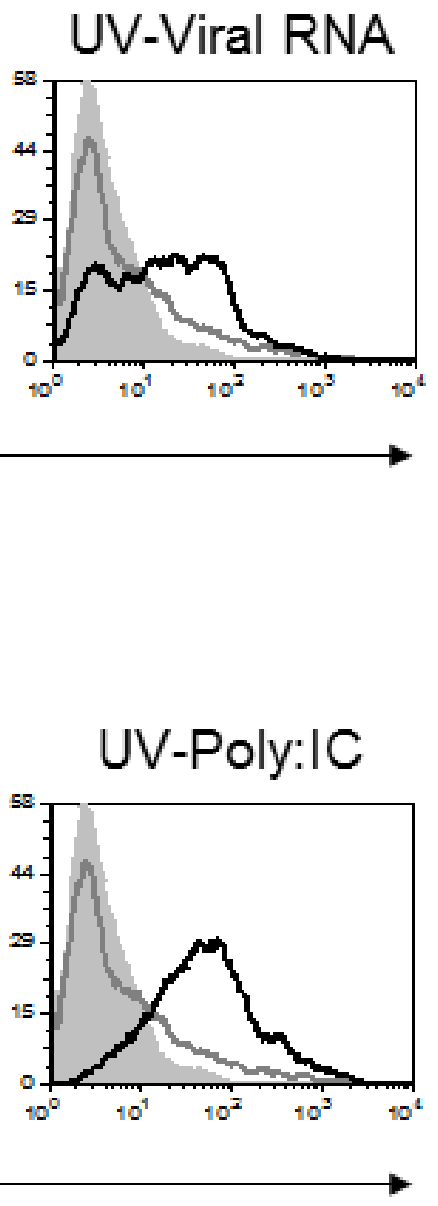

Figure 3: Induction of CEACAM1 on A549 cells following RNA treatments. a. Analysis of CEACAM1 expression on A549 cells, 3 days post infection. The A549 cells were infected either with HMPV (left) or with UV-inactivated virus (right, UV-HMPV). b. A549 cells were transfected with HMPV RNA (Viral RNA) or with HMPV RNA that underwent UV inactivation (UV-Viral RNA). (c) A549 cells were transfected either with Poly:IC (left) or with Poly:IC that was inactivated by UV treatment (UV-Poly:IC). For all figure parts CEACAM1 staining of the treated cells is represented by the black empty histograms. The background staining is represented by the gray filled histograms and mock CEACAM1 staining is the gray empty histograms. The background staining and the CEACAM1 staining of the MOCK cells is identical in all figure parts a.-c. and is shown several times for clarity. The background staining of the various treatment was similar to the MOCK treatment and is not shown in the figure. Figure shows one representative experiment out of two independent experiments. 
a

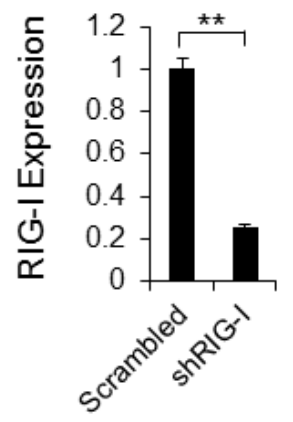

b

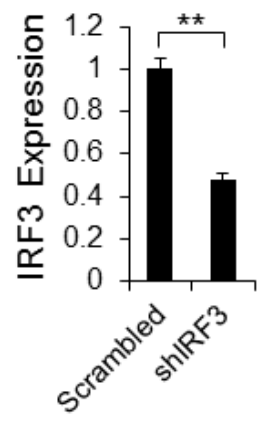

C

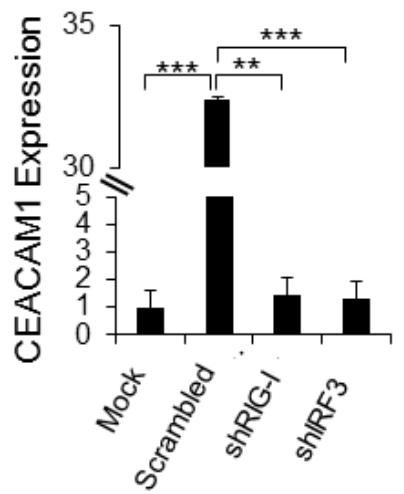

Figure 4: CEACAM1 induction is mediated by RIG-I and IRF3. a.-b. qRT-PCR quantification of RIG-I (a) and IRF3 (b) expression in A549 cells that underwent IRF3 and RIG-I targeted shRNA knockdowns compared with the same cells transduced with scrambled shRNA (Scrambled set as 1). c. The scrambled A549 cells and the specific shRNA knockdown cells from (a) and (b) were infected with hMPV and analyzed by FACS for induction of CEACAM1 on mock (Mock) cells was set up to be 1. Figure shows one representative result out of three independent experiments. ${ }^{* *} P<0.01,{ }^{* * *} P<0.001$.

a

\section{IRF3 binding site}

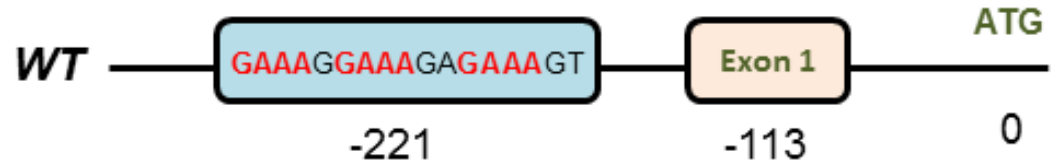

b

IRF3 binding site

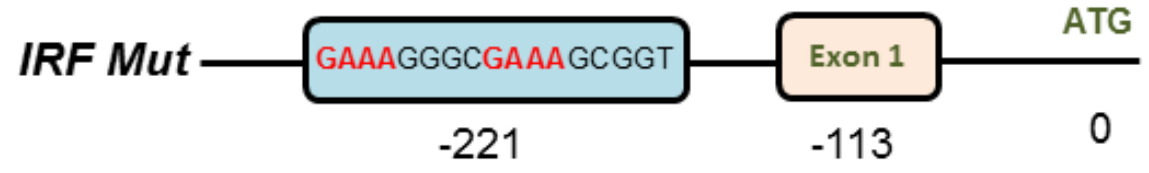

C

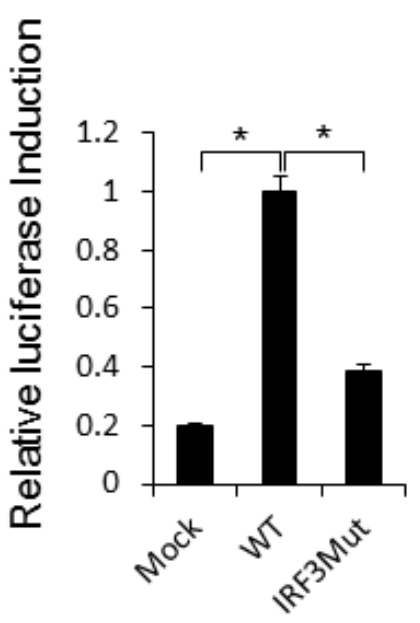

Figure 5: Direct IRF3 mediated induction of CEACAM1 during HMPV infection. a.-b. Schematic representation of the CEACAM1 promoter region with "Exon1" indicating transcript initiation point and "ATG" the start of the CEACAM1 open reading frame (ORF). The wild-type IRF3 binding site sequence is indicated (a) along with the mutation introduced into the IRF3 site (IRF3 Mut, b). c. Fold increase in luciferase activity in A549 cells. Mock or HMPV infected A549 cells were transfected with luciferase encoding vector fused downstream to the wild-type CEACAM1 promoter (WT), or luciferase fused to the mutated CEACAM1 promoter (IRF3M). The figure shows data from three experiments combined. Statistical analysis was calculated on the data from all experiments combined. $* p<$ 0.03 . 


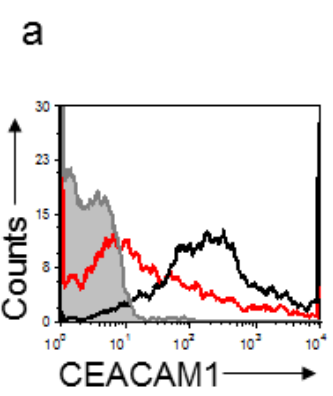

e

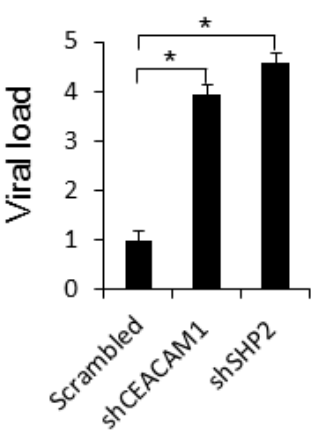

$\mathrm{h}$

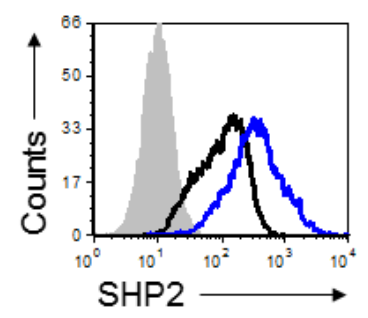

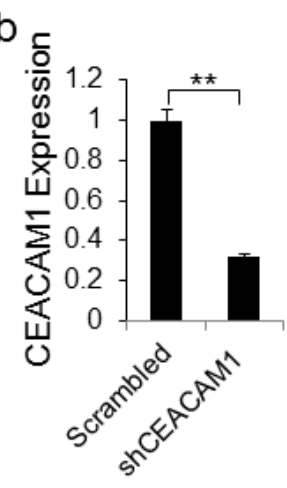

f
C

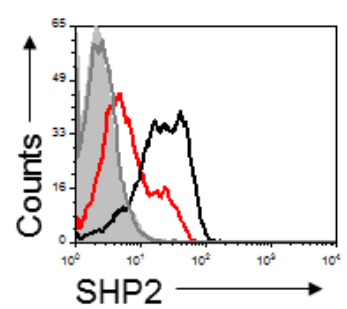

d

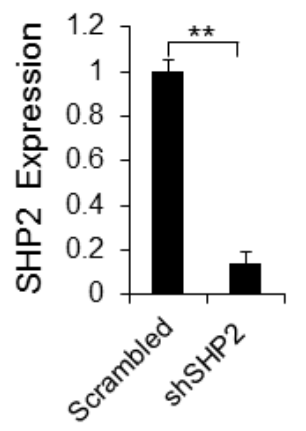

g
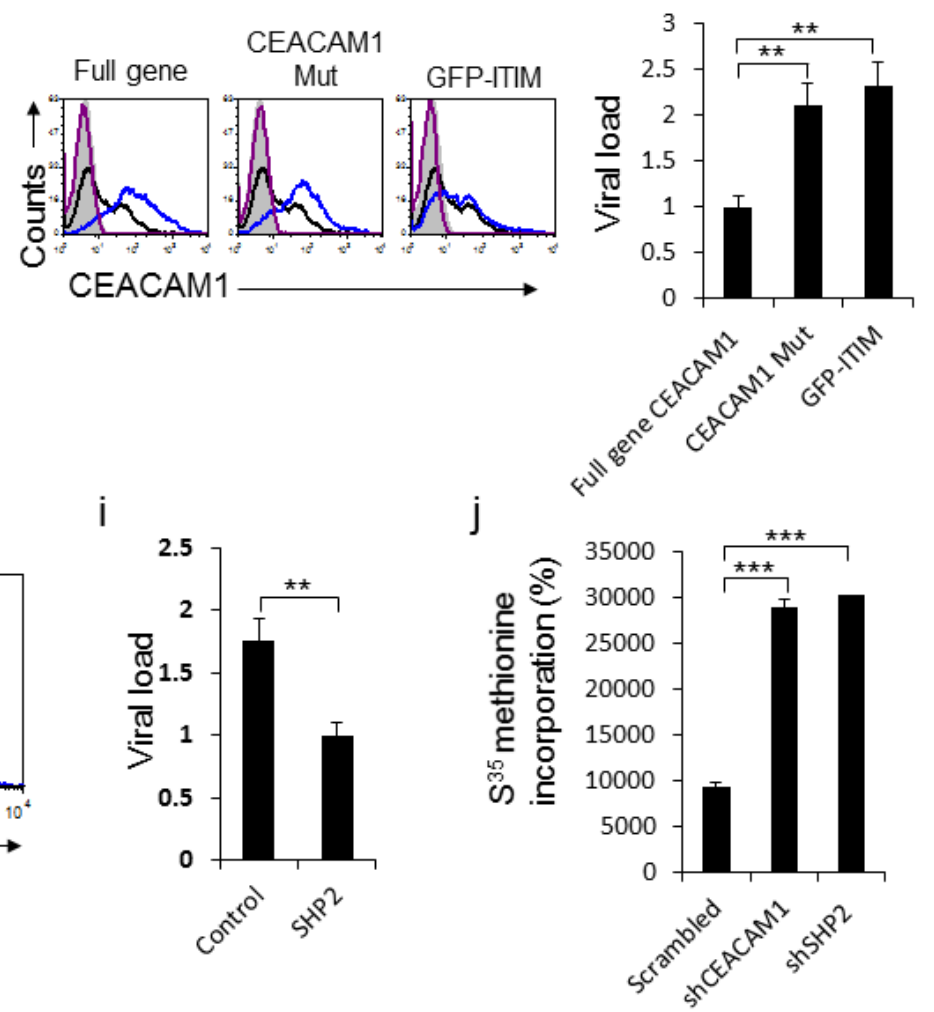

Figure 6: CEACAM1 inhibits HMPV infection through SHP2. a. CEACAM1 was knockdown in A549 cells using specific shRNA (red histogram), scrambled shRNA was used as control (black empty histogram). The gray empty histogram and the gray filled histogram represent the staining with control Ab of A549 cells transduced with shRNA against CEACAM1 and scrambled shRNA, respectively. b. qRT-PCR quantification of CEACAM1 down regulation following the shRNA knockdown. A549 cells transduced with scrambled was set up as 1. c. SHP2 was knockdown on A549 cells using specific shRNA (red histogram), scrambled shRNA was used as control (black empty histogram). The gray empty histogram and the gray filled histogram represent the staining with control Ab of A549 cells transduced with shRNA against SHP2 and scrambled shRNA, respectively. d. Real time PCR quantification of SHP2 down regulation following the shRNA knockdown of SHP2. A549 cells transduced with Scrambled was set up as 1. e. Quantification of viral loads in the supernatants of A549 infected cells expressing CEACAM1 or SHP2 specific shRNAs compared to the scrambled (Scrambled, set as 1). f. FACS analysis of the overexpressed full CEACAM1 gene, CEACAM1 lacking the intracellular domain (CEACAM1 Mut) or a construct contain the intracellular domain fused to GFP (GFP-ITIM), showed as blue empty histograms. Control expression of CEACAM1 on untreated A549 cells (black empty histogram). The purple empty histogram and the gray filled histogram represent the staining with control $\mathrm{Ab}$ of A549 cells overexpressed with full gene CEACAM1 or CEACAM1 mutants and untreated cells, respectively. g. Quantification of viral loads in the supernatants of A549 infected cells expressing the full gene of CEACAM1, (CEACAM1 Mut) or (GFP-ITIM). The full gene CEACAM1, set as 1. h. SHP2 overexpression in A549 cells (blue empty histogram), as compared to control SHP2 expression (black empty histogram). The gray filled histogram represent the staining with control Ab of A549 cells over expressing with SHP2. The control staining of parental cells was similar and is not shown in the figure. i. Quantification of viral loads in the supernatants of A549 infected cells overexpressing SHP2 compared to the control. The overexpressed SHP2, set as 1. j. A549 cells stably expressing shCEACAM1 or shSHP2 were analyzed for total protein production by assessing the rate of ${ }^{35} \mathrm{~S}$-Methionine incorporation compared to the scrambled. The figure shows data from two experiments combined. ${ }^{*} P<0.05 ; * * P<0.01 ; * * * P<0.001$. 
The mechanism underlying the CEACAM1mediated suppression involves inhibition of the cellular translation machinery, can be detrimental to healthy cells. However, we demonstrate that CEACAM1 induction is restricted to the HMPV-infected cells. We propose that the innate immune system developed this specific mechanism that harnesses CEACAM1 as an anti-viral mechanism, unique properties and due to its homophilic interactions. Thus, through the induction of CEACAM1 only, virus production is inhibited.

\section{MATERIALS AND METHODS}

\section{Viruses and cell lines}

Vero, kidney epithelial cells derived from an African green monkey, and A549, human alveolar type II-like epithelial cells, were maintained in Dulbecco's modified Eagle medium (DMEM) with standard supplements and
$10 \%$ fetal calf serum (FCS). To propagate HMPV, around $70 \%$ confluent Vero cells were infected with recombinant (HMPV-GFP) strains as previously described [37] using MOI 0.1 in medium containing $0.25 \mathrm{mg} / \mathrm{ml}$ trypsin for one hour. DMEM medium with standard supplements containing 3\% FCS was then added. Five days after infection, cells were scraped from the plates, collected, freeze-thawed $\left(-80^{\circ} \mathrm{C} / 37^{\circ} \mathrm{C}\right)$ three times and crude viruses were stored at $-80^{\circ} \mathrm{C}$. Viral loads were determined by using quantitative RT-PCR (qRT-PCR) and/or by using fluorescence microscopy. For A549 infection experiments, around $70 \%$ confluent cells were infected with HMPV, in serum-free media with $0.25 \mathrm{mg} / \mathrm{ml}$ trypsin for 1 hour incubation at $37^{\circ} \mathrm{C}$, then maintained in DMEM medium with standard supplements containing $2 \%$ FCS.

\section{Viral RNA isolation and transfections}

For preparation of shRNA, 293T cells were cotransfected with the lentiviral vector containing the

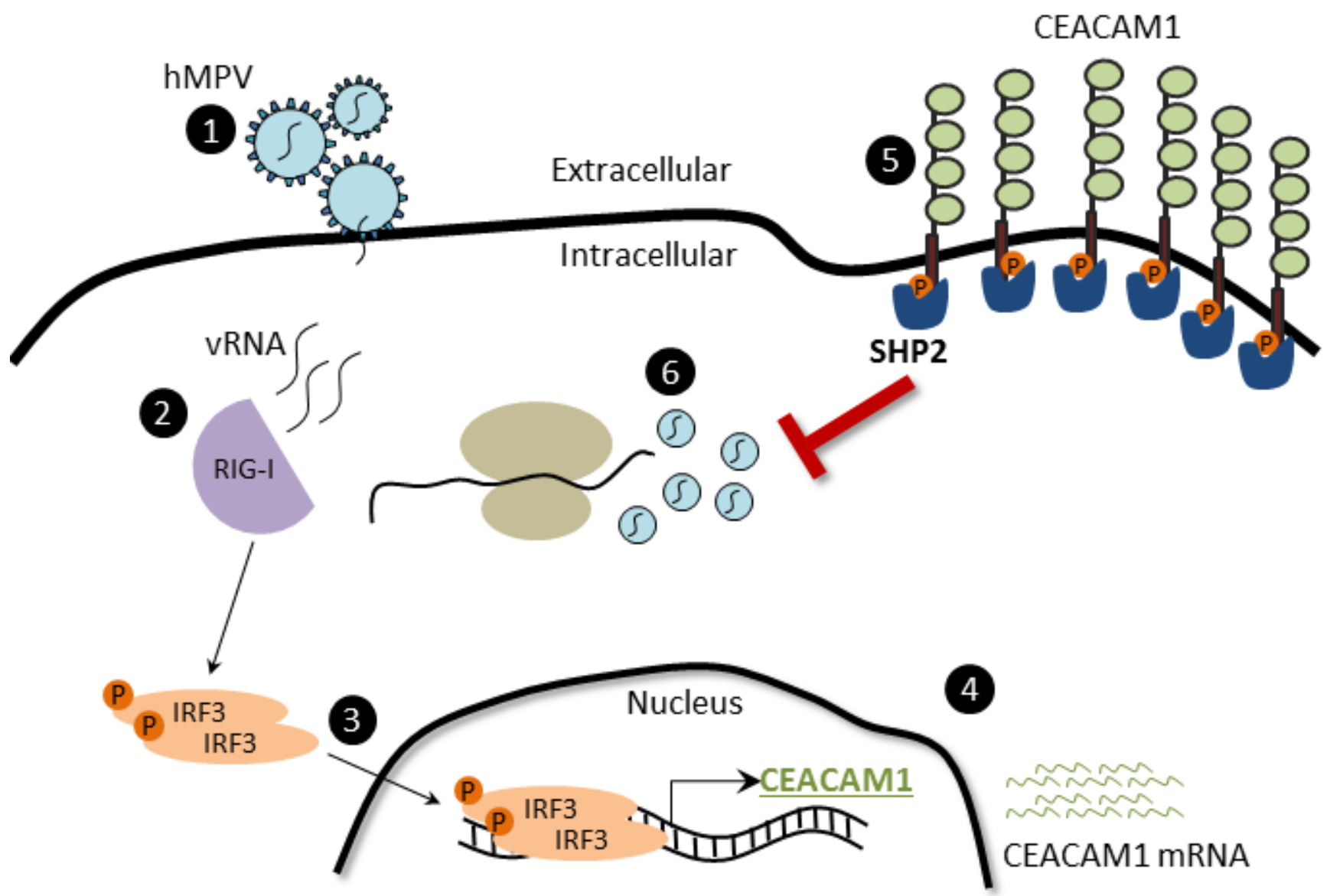

Figure 7: The CEACAM1-mechanism of action during hMPV infection. (1) HMPV binds and infects the cells. (2) HMPV RNA is sense by RIG-I, which subsequently activates downstream the phosphorylation of IRF3. (3) IRF3 undergoes conformational changes and dimerization, then translocates to the nucleus and binds the CEACAM1 promoter. (4) CEACAM1 mRNA is transcribed. (5) CEACAM1 is overexpressed on the cell surface of infected cells. (6) The SHP2 phosphatase anchor on CEACAM1 ITIMs and subsequently inhibits the HMPV replication by inhibiting protein production. 
shRNA, a plasmid encoding the lentiviral Gag/Pol, and a plasmid encoding the VSV-G at a 10:6.5:3.5 ratios respectively. Supernatants contaning the viral particles were collected after 48 hours. A549 cells were selected for puromycin resistance at $5 \mu \mathrm{g} / \mathrm{ml}$. Viral RNA that was transfected was isolated from cell free purified viral stocks by easyMag system (BioMerieux). Viral RNA transfection was performed on cells that were plated in 24 well plates, at $50 \mathrm{~K}$ cells/well, which were subsequently transfected with $1 \mu \mathrm{g} / \mathrm{ml}$ of DNA with $2 \mu \mathrm{l} / \mu \mathrm{g}$ of LT- 1 (MirusBio) transfection reagent per DNA, according to manufacturer's recommendations. UV inactivation prior to viral infection or viral RNA transfection, was performed with the UV Stratalinker 2400 (StrataGene) at 0.99 Joule.

\section{FACS staining}

Staining for CEACAM1 was conducted by FACS staining for human CEACAM1 with anti-CEACAM1 $\mathrm{mAb}$ (Biolegend). Intracellular staining for SHP2 was conducted using an anti-SHP2 antibody (Santa Cruz). Intracellular FACS staining for SHP2 was based on methanol fixation protocol. Briefly, cells were perforated in methanol at $-20^{\circ} \mathrm{C}$, rehydrated in PBS for half an hour, and then stained with antibodies in 5\% BSA in PBS at room temperature.

\section{Cloning, qRT-PCR and shRNAs}

Stable transduction of CEACAM1, CEACAM1Mut, GFP-ITIM and SHP2 transfectants was based on lentiviral and retroviral expression systems. For mRNA quantification, total RNA was isolated from cells using the Total RNA isolation kit (Zymoresearch). RNA was reverse transcribed with Moloney murine leukemia virus reverse transcriptase (Invitrogen) and with polyT primer (Sigma). Quantitative amplification was conducted on an ABI PRISM 7900 real-time PCR system (Applied Biosystems) with gene specific primers and Platinum SYBR Green qPCR SuperMix-UDG with ROX (Invitrogen). Newly synthesized transcript analysis was performed as previously described [38]. RNA was reverse transcribed and analyzed by real-time PCR. Primers used for the realtime PCR:

Amplification of hCEACAM1 long variant only fwd 5'TGCTGAACGTAAACTATAATGCTCT, rev 5' GGAGACTGAGGGTTTGTGCT [39].

Amplification of HMPV nucleoprotein gene $(\mathrm{N})$

Forward (fwd) 5,

CATATAAGCATGCTATATTAAAAGAGTCTC, rev 5'

CCTATTTCTGCAGCATATTTGTAATCAG

IRF3 fwd 5'GATGCACAGCAGGAGGATTT, rev

5' TAAACGCAACCCTTCTTTGC;

RIG-I fwd 5'ATCCCAGTGTATGAACAGCAG; rev 5'GCCTGTAACTCTATACCCATGTC. SHP2 fwd GGGTGGAGACACGACACTTT; rev 5' GGTTCTTCACCAAGCTGGAC

HPRT and GAPDH used as normalizers for qPCR analyses. HPRT fwd 5'TGACACTGGCAAAACAATGCA; rev 5'GGTCCTTTTCACCAGCAAGCT GAPDH fwd 5'TGCACCACCAACTGCTTA; rev 5'GGATGCAGGGATGATGTTC. Primers used for cloning and mutating CEACAM1 promoter (cloned with XhoI and HindIII) in pGL4.14 firefly luciferase expression vector (Promega) fwd 5'CGCCTCGAGCCTGGACTTGGGTCTCTGTC; Mutation reversed (rev) 5, TCCTACCTTTGTC CTTACCGCTTTCGCCCTTTCTGTCTACATTTT; Mutation fwd 5, AAAATGTAGACAGAA AGG CGAAAGCGGTAAGGACAAAGTAGGA;

CEACAM1 promoter, rev 5'CGCAAGCTTTCACCTGTGGAGGAGAGCTT.

shRNA sequences were based on the pLKO.1 lentiviral vector backbone with puromycin selection marker (Sigma).

IRF3
5' CCGGGATCTGATTACCTTCACGGAAC
TCGAGTTCCGTGAAGGTAATCAGATCTTTTT.
RIG-I
5'CCGGCCATGTGAAGTACAAGACATTCTC
GAGAATGTCTTGTACTTCACATGGTTTTTTG.
CEACAM1
5'CCGGCCACCTAACAAGATGAATGAAC
TCGAGTTCATTCATCTTGTTAGGTGGTTTTT.
SHP2
5'CCGGGCAGTTAAATTGTGCGCTGTACT
CGAGTACAGCGCACAATTTAACTGCTTTTT.

\section{BW assay}

BW transfected cells were prepared as described elsewhere [40]. 50,000 of the appropriate BW or BW transfectants were incubated together with equal numbers of irradiated (6000 rad) mock-infected or HMPV-infected A549 cells for 48 hours at $37^{\circ} \mathrm{C}$ and $5 \% \mathrm{CO} 2$. Following 48 hours incubation, the presence of mouse IL-2 in the supernatants was determined using standard enzymelinked immunosorbent assay (ELISA). Student's t test was used to determine significant differences.

\section{Luciferase assay}

Cells were grown to $50 \%-60 \%$ confluence in 24 well plates. Cells were then

transfected using LT-1 transfection reagent (MirusBio), with 60ng of the respective firefly luciferase reporter vector pGL4.14 and 5ng of control vector, encoding Renilla luciferase, pRL-CMV (Promega), in 
a final volume of $0.5 \mathrm{ml}$. Firefly and Renilla luciferase activities were then measured consecutively using the Dual-luciferase assays (Promega), $48 \mathrm{hr}$ after transfection.

\section{Protein translation assay}

A549 cells grown in 24-well replicates were resuspended in methinonine-free DMEM based (Sigma) medium. Following methionine starvation, labeled $\left[{ }^{35} \mathrm{~S}\right]$ Methionine was supplemented to the medium to a final labeling concentration of $0.05 \mu \mathrm{Ci} / \mu \mathrm{L}$ for 5 hours of incubation. Cells were then washed with PBS, lysed in $1 \mathrm{ml}$ of $\mathrm{NaOH} 0.1 \mathrm{M}$, supplemented with $1: 3$ ration of scintillation liquid and analyzed by $\beta$-Counter.

\section{Statistical analysis}

Statistical significance was determined by Students t-test. P value of less than 0.05 was considered significant and indicated in figures and figure legends.

\section{ACKNOWLEDGMENTS}

We thank Prof. Ron Fouchier (Erasmus Medical Center, Netherlands) for providing the reverse genetics system for HMPV.

\section{GRANT SUPPORT}

This study was supported by the European Research Council under the European Union's Seventh Framework Programme (FP/2007-2013) / ERC Grant Agreement number 320473-BacNK. Further support came from the Israel Science Foundation, the GIF foundation, the Lewis family foundation, the ICRF professorship grant, the Helmholtz Israel grant and the Rosetrees Trust (all to O.M.).

\section{CONFLICTS OF INTEREST}

There is no conflict of interest.

\section{REFERENCES}

1. van den Hoogen BG, de Jong JC, Groen J, Kuiken T, de Groot R, Fouchier RA and Osterhaus AD. A newly discovered human pneumovirus isolated from young children with respiratory tract disease. Nature medicine. 2001; 7:719-724.

2. Williams JV, Harris PA, Tollefson SJ, Halburnt-Rush LL, Pingsterhaus JM, Edwards KM, Wright PF and Crowe JE, Jr. Human metapneumovirus and lower respiratory tract disease in otherwise healthy infants and children. The New England journal of medicine. 2004; 350:443-450.
3. Williams JV, Wang CK, Yang CF, Tollefson SJ, House FS, Heck JM, Chu M, Brown JB, Lintao LD, Quinto JD, Chu D, Spaete RR, Edwards KM, Wright PF and Crowe JE, Jr. The role of human metapneumovirus in upper respiratory tract infections in children: a 20-year experience. The Journal of infectious diseases. 2006; 193:387-395.

4. van den Hoogen BG, van Doornum GJ, Fockens JC, Cornelissen JJ, Beyer WE, de Groot R, Osterhaus AD and Fouchier RA. Prevalence and clinical symptoms of human metapneumovirus infection in hospitalized patients. The Journal of infectious diseases. 2003; 188:1571-1577.

5. Peiris JS, Tang WH, Chan KH, Khong PL, Guan Y, Lau YL and Chiu SS. Children with respiratory disease associated with metapneumovirus in Hong Kong. Emerging infectious diseases. 2003; 9:628-633.

6. Mullins JA, Erdman DD, Weinberg GA, Edwards K, Hall CB, Walker FJ, Iwane M and Anderson LJ. Human metapneumovirus infection among children hospitalized with acute respiratory illness. Emerging infectious diseases. 2004; 10:700-705.

7. Mackay IM, Bialasiewicz S, Jacob KC, McQueen E, Arden KE, Nissen MD and Sloots TP. Genetic diversity of human metapneumovirus over 4 consecutive years in Australia. The Journal of infectious diseases. 2006; 193:1630-1633.

8. Dollner H, Risnes K, Radtke A and Nordbo SA. Outbreak of human metapneumovirus infection in norwegian children. The Pediatric infectious disease journal. 2004; 23:436-440.

9. Williams JV, Martino R, Rabella N, Otegui M, Parody R, Heck JM and Crowe JE, Jr. A prospective study comparing human metapneumovirus with other respiratory viruses in adults with hematologic malignancies and respiratory tract infections. The Journal of infectious diseases. 2005; 192:1061-1065.

10. Larcher C, Geltner C, Fischer H, Nachbaur D, Muller LC and Huemer HP. Human metapneumovirus infection in lung transplant recipients: clinical presentation and epidemiology. The Journal of heart and lung transplantation. 2005; 24:1891-1901.

11. Williams JV, Crowe JE, Jr., Enriquez R, Minton P, Peebles RS, Jr., Hamilton RG, Higgins S, Griffin M and Hartert TV. Human metapneumovirus infection plays an etiologic role in acute asthma exacerbations requiring hospitalization in adults. The Journal of infectious diseases. 2005; 192:11491153.

12. Madhi SA, Ludewick H, Abed Y, Klugman KP and Boivin G. Human metapneumovirus-associated lower respiratory tract infections among hospitalized human immunodeficiency virus type 1 (HIV-1)-infected and HIV1-uninfected African infants. Clinical infectious diseases. 2003; 37:1705-1710.

13. Bastien N, Ward D, Van Caeseele P, Brandt K, Lee $\mathrm{SH}$, McNabb G, Klisko B, Chan E and Li Y. Human metapneumovirus infection in the Canadian population. Journal of clinical microbiology. 2003; 41:4642-4646. 
14. Hamelin ME, Cote S, Laforge J, Lampron N, Bourbeau J, Weiss K, Gilca R, DeSerres G and Boivin G. Human metapneumovirus infection in adults with communityacquired pneumonia and exacerbation of chronic obstructive pulmonary disease. Clinical infectious diseases. 2005; 41:498-502.

15. Garcia-Garcia ML, Calvo C, Casas I, Bracamonte T, Rellan A, Gozalo F, Tenorio T and Perez-Brena P. Human metapneumovirus bronchiolitis in infancy is an important risk factor for asthma at age 5. Pediatric pulmonology. 2007; 42:458-464.

16. Kahn JS. Epidemiology of human metapneumovirus. Clinical microbiology reviews. 2006; 19:546-557.

17. Panda S, Mohakud NK, Pena L and Kumar S. Human metapneumovirus: review of an important respiratory pathogen. International journal of infectious diseases. 2014; 25:45-52.

18. Seth RB, Sun L and Chen ZJ. Antiviral innate immunity pathways. Cell research. 2006; 16:141-147.

19. Meylan E, Tschopp J and Karin M. Intracellular pattern recognition receptors in the host response. Nature. 2006; 442:39-44.

20. Kawai $\mathrm{T}$ and Akira $\mathrm{S}$. The role of pattern-recognition receptors in innate immunity: update on Toll-like receptors. Nature immunology. 2010; 11:373-384.

21. Kumar H, Kawai $\mathrm{T}$ and Akira S. Toll-like receptors and innate immunity. Biochemical and biophysical research communications. 2009; 388:621-625.

22. Sabbah A, Chang TH, Harnack R, Frohlich V, Tominaga $\mathrm{K}$, Dube PH, Xiang Y and Bose S. Activation of innate immune antiviral responses by Nod2. Nature immunology. 2009; 10:1073-1080.

23. Loo YM, Fornek J, Crochet N, Bajwa G, Perwitasari O, Martinez-Sobrido L, Akira S, Gill MA, Garcia-Sastre A, Katze MG and Gale M, Jr. Distinct RIG-I and MDA5 signaling by RNA viruses in innate immunity. Journal of virology. 2008; 82:335-345.

24. Yoneyama M, Onomoto K, Jogi M, Akaboshi T and Fujita T. Viral RNA detection by RIG-I-like receptors. Current opinion in immunology. 2015; 32:48-53.

25. Bao X, Liu T, Spetch L, Kolli D, Garofalo RP and Casola A. Airway epithelial cell response to human metapneumovirus infection. Virology. 2007; 368:91-101.

26. Goutagny N, Jiang Z, Tian J, Parroche P, Schickli J, Monks BG, Ulbrandt N, Ji H, Kiener PA, Coyle AJ and Fitzgerald KA. Cell type-specific recognition of human metapneumoviruses (HMPVs) by retinoic acid-inducible gene I (RIG-I) and TLR7 and viral interference of RIG-I ligand recognition by HMPV-B1 phosphoprotein. Journal of immunology. 2010; 184:1168-1179.

27. Spann KM, Loh Z, Lynch JP, Ullah A, Zhang V, Baturcam E, Werder RB, Khajornjiraphan N, Rudd P, Loo YM, Suhrbier A, Gale M, Jr., Upham JW and Phipps S. IRF-3,
IRF-7, and IPS-1 promote host defense against acute human metapneumovirus infection in neonatal mice. The American journal of pathology. 2014; 184:1795-1806.

28. Hiscott J. Triggering the innate antiviral response through IRF-3 activation. The Journal of biological chemistry. 2007; 282:15325-15329.

29. Liao S, Bao X, Liu T, Lai S, Li K, Garofalo RP and Casola A. Role of retinoic acid inducible gene-I in human metapneumovirus-induced cellular signalling. The Journal of general virology. 2008; 89:1978-1986.

30. Obrink B. CEA adhesion molecules: multifunctional proteins with signal-regulatory properties. Current opinion in cell biology. 1997; 9:616-626.

31. Beauchemin N, Draber P, Dveksler G, Gold P, Gray-Owen S, Grunert F, Hammarstrom S, Holmes KV, Karlsson A, Kuroki M, Lin SH, Lucka L, Najjar SM, et al. Redefined nomenclature for members of the carcinoembryonic antigen family. Experimental cell research. 1999; 252:243-249.

32. Gray-Owen SD and Blumberg RS. CEACAM1: contact-dependent control of immunity. Nature reviews Immunology. 2006; 6:433-446.

33. Watt SM, Teixeira AM, Zhou GQ, Doyonnas R, Zhang Y, Grunert F, Blumberg RS, Kuroki M, Skubitz KM and Bates PA. Homophilic adhesion of human CEACAM1 involves $\mathrm{N}$-terminal domain interactions: structural analysis of the binding site. Blood. 2001; 98:1469-1479.

34. Stern N, Markel G, Arnon TI, Gruda R, Wong H, GrayOwen SD and Mandelboim O. Carcinoembryonic antigen (CEA) inhibits NK killing via interaction with CEA-related cell adhesion molecule 1. Journal of immunology. 2005; 174:6692-6701.

35. Nouvion AL, Oubaha M, Leblanc S, Davis EC, Jastrow H, Kammerer R, Breton V, Turbide C, Ergun S, Gratton JP and Beauchemin N. CEACAM1: a key regulator of vascular permeability. Journal of cell science. 2010; 123:4221-4230.

36. Muller MM, Klaile E, Vorontsova O, Singer BB and Obrink B. Homophilic adhesion and CEACAM1-S regulate dimerization of CEACAM1-L and recruitment of SHP-2 and c-Src. The Journal of cell biology. 2009; 187:569-581.

37. Herfst S, de Graaf M, Schickli JH, Tang RS, Kaur J, Yang CF, Spaete RR, Haller AA, van den Hoogen BG, Osterhaus $\mathrm{AD}$ and Fouchier RA. Recovery of human metapneumovirus genetic lineages a and B from cloned cDNA. Journal of virology. 2004; 78:8264-8270.

38. Halenius A, Hauka S, Dolken L, Stindt J, Reinhard H, Wiek C, Hanenberg H, Koszinowski UH, Momburg F and Hengel $\mathrm{H}$. Human cytomegalovirus disrupts the major histocompatibility complex class I peptide-loading complex and inhibits tapasin gene transcription. Journal of virology. $2011 ; 85: 3473-3485$.

39. Maertzdorf J, Wang CK, Brown JB, Quinto JD, Chu M, de Graaf M, van den Hoogen BG, Spaete R, Osterhaus AD and Fouchier RA. Real-time reverse transcriptase PCR assay for detection of human metapneumoviruses from all known 
genetic lineages. Journal of clinical microbiology. 2004; 42:981-986.

40. Achdout H, Arnon TI, Markel G, Gonen-Gross T, Katz G, Lieberman N, Gazit R, Joseph A, Kedar E and Mandelboim O. Enhanced recognition of human NK receptors after influenza virus infection. Journal of immunology. 2003; 171:915-923.

41. Sabo Y, Ehrlich M and Bacharach E. The conserved YAGL motif in human metapneumovirus is required for higherorder cellular assemblies of the matrix protein and for virion production. Journal of virology. 2011; 85:6594-6609.
42. Eisenacher K and Krug A. Regulation of RLR-mediated innate immune signaling - it is all about keeping the balance. European journal of cell biology. 2012; 91:36-47.

43. Vitenshtein A, Weisblum Y, Hauka S, Halenius A, OiknineDjian E, Tsukerman P, Bauman Y, Bar-On Y, SternGinossar N, Enk J, Ortenberg R, Tai J, Markel G, et al. CEACAM1-Mediated Inhibition of Virus Production. Cell reports. 2016; 15:2331-2339. 